\title{
NMR Observation of Entangled Polymer Dynamics: Tube Model Predictions and Constraint Release
}

\author{
Fabián Vaca Chávez and Kay Saalwächter* \\ Institut für Physik-NMR, Martin-Luther-Universität Halle-Wittenberg, Betty-Heimann-Straße 7, D-06120 Halle, Germany
}

(Received 25 January 2010; published 12 May 2010)

\begin{abstract}
We use a benchtop NMR technique to monitor entangled polymer melt dynamics over 5 decades in time covering regimes II-IV of the tube model. We confirm the familiar molar mass scaling exponents of the regime transitions, but we observe a mass-dependent time scaling exponent describing segmental fluctuations in the constrained-Rouse regime II up to high molecular weights. Local chain motions are thus governed by modes that are much less restricted than predicted by the tube model. Diluting protonated chains in deuterated invisible matrix chains, we prove that the exponent is determined by the matrix molecular weight, suggesting constraint-release effects as the origin.
\end{abstract}

DOI: 10.1103/PhysRevLett.104.198305

PACS numbers: $83.80 . \mathrm{Sg}, 76.60 .-\mathrm{k}, 83.10 . \mathrm{Kn}$

The tube model of polymer dynamics [1] provides a molecular rationale of the fascinating mechanical behavior of long-chain polymer melts. It combines a bead-spring (Rouse) model for unentangled chains with de Gennes' reptation model [2], which assumes a snakelike large-scale motion along a confining tube, thus explaining transient elasticity and long-term flow. However, the simple fixedtube model is insufficient for the quantitative description of rheological data, and ongoing discussions consider dynamics of the tube itself, caused by contour-length fluctuations (CLF), arising from chain-end motions of the test chain, or constraint release (CR), arising from matrix chain motions [3-5]. Microscopic, atomic-scale experiments focusing on the Doi-Edwards regimes II-IV (constrained Rouse, reptation, and terminal diffusion) are the only way to crucially test the fundamental model assumptions, and dielectric [6], neutron spin-echo (NSE) [7] and various NMR spectroscopy techniques [8-15] have been applied for that purpose.

Information on detailed characteristics of the chain motion below and above the Rouse time $\tau_{R}$ and on the validity of the fixed-tube reptation picture in well-entangled melts still remains scarce. This work demonstrates the quantitative molecular observation of the scaling laws in regimes II and III, and the measurement of the Rouse time $\tau_{R}$ and the disentanglement time $\tau_{d}$ in long-chain melts. We observe characteristic deviations from the tube-model predictions up to high molecular weights, and show that CR processes are responsible for modified chain modes faster than actual reptation. Our results extend previous observations of the segmental mean-squared displacement $\left\langle\Delta r^{2}\right\rangle$ by NSE spectroscopy [7] and diffusion NMR [8-11], where the limited dynamic range and immaterial spin diffusion [11], respectively, pose challenges to the study of well-entangled bulk systems. We use ${ }^{1} \mathrm{H}$ multiple-quantum (MQ) NMR $[12,16]$, which measures segmental dynamics in terms of an orientation autocorrelation function of the second Legendre polynomial $C(t)=\left\langle P_{2}(\cos \theta(t)) P_{2}(\cos \theta(0))\right\rangle$, where $\theta$ is the segmental orientation relative to the external magnetic field. While the tube model predicts power laws for $\left\langle\Delta r^{2}\right\rangle$ following $t^{1 / 4}, t^{1 / 2}$, and $t^{1}$ in regimes II to IV, $C(t)$ as derived by Ball et al. [17] should exhibit exponents $-1 / 4$ and $-1 / 2$ below (II) and above $\tau_{R}$ (III), respectively. Regime III is further characterized by a smooth downturn in a log-log plot, starting with a slope of $-1 / 2$ and reaching $-3 / 2$ at $t=\tau_{d}$, followed by complete loss of correlation in regime IV.

Many NMR relaxation techniques are sensitive to $C(t)$, and an advantage is the sensitivity to molecular rotations over many decades in time, such that also slow processes not involving significant displacement can be studied. This offers unique possibilities for the investigation of dynamics in complex topologies such as branched structures or brushes. NMR $T_{1}$ relaxation dispersion experiments by field cycling have recently gained momentum [13-15]. In a recent paper, Herrmann et al. [15] demonstrated the measurement of $C(t)$ for linear poly(butadiene), $\mathrm{PB}$, over 8 decades in time, starting with the $\alpha$ processes in a "regime 0," and covering the transition from the free Rouse regime I to entangled dynamics, but observing only a regime-II exponent of $-1 / 2$ rather than $-1 / 4$ at the highest molar mass $M$ of $18 \mathrm{kDa}$.

In contrast to the mentioned relaxation methods, MQ NMR is based on fundamentally different principles, measuring a time integral of correlated dipolar couplings, thus $C(t)$, directly and model free in the time domain [12] rather than deriving it from a spectral density, which has to be known for all frequencies in order to obtain $C(t)$ as its Fourier transform. We improved upon the magic-angle spinning method of Graf et al. [12], which is subject to systematic errors related to an ill-defined absolute intensity and the impossibility to realize sufficiently short evolution times. A simple static version [16], robust enough for a benchtop spectrometer, provides a smooth and artifact-free measure of $C(t)$.

The major observable is a normalized intensity buildup related to double-quantum (DQ) coherences, $I_{\mathrm{nDQ}}\left(\tau_{\mathrm{DQ}}\right)$, 
measured as a function of the pulse sequence time $\tau_{\mathrm{DQ}}$ on a scale relative to full magnetization of entangled segments. It depends on the magnitude of the temperature(thus time-) dependent apparent residual dipolar coupling, $D_{\text {res }}$, which arises from anisotropic fluctuations of the monomer-fixed dipole-dipole coupling tensors. The data analysis involves the subtraction of a temperaturedependent, well-separated slowly decaying signal fraction [16], which in a linear melt is related to unentangled, isotropically moving chain ends. It arises from CLF effects [18] and contributes to a decrease in elasticity by diluting the entangled fraction. In the short- $\tau_{\mathrm{DQ}}$ limit, the $I_{\mathrm{nDQ}}\left(\tau_{\mathrm{DQ}}\right)$ associated with entangled center segments was shown to be directly related to $C\left(t>\tau_{e}\right)=C\left(\tau_{e}\right) \times$ $C_{e}\left(t>\tau_{e}\right)[12]$,

$$
I_{\mathrm{nDQ}}\left(\tau_{\mathrm{DQ}}>\tau_{e}\right)=A S_{e}^{2} \tau_{\mathrm{DQ}}^{2} C_{e}\left(\tau_{\mathrm{DQ}}\right) \propto D_{\mathrm{res}}^{2} \tau_{\mathrm{DQ}}^{2},
$$

where $S_{e}=\sqrt{C\left(\tau_{e}\right)} \approx 3 / 5 N_{e}$ is the entanglement-induced dynamic order parameter describing the local orientation of segments, depending on the number of statistical (Kuhn) segments in an entangled unit $N_{e}$.

We study monodisperse PB (-H and -sec-butyl termination, $\mathrm{PD} \leq 1.05$, about $54 \%$ cis, $41 \%$ trans, and $5 \%$ vinyl units, purchased at PSS, Mainz, Germany), referred to as $\mathrm{PB} x$ where $x$ is the molecular weight in Da. We note the number of entangled units $Z=M / M_{e}$, with $M_{e}=$ $1.85 \mathrm{kDA}$ [19]. The sample data for the highest $M$ sample PB2000k in Fig. 1(a) demonstrate the effect of regime-II motion: $C\left(\tau_{\mathrm{DQ}}\right)$ is progressively reduced at higher temperatures, delaying the buildup. At $243 \mathrm{~K}$ and below, local order is too large and the intensities become challenged by signal loss related to limitations posed by the pulse sequence, rendering the long-time data noisy. The curve for $303 \mathrm{~K}$ has the best signal-to-noise and still reaches a high level of intensity, not far from the expected plateau value of 0.5 . The plateau value is in fact realized for networks, i.e., without reptation.

Only the absolute-value of $C\left(\tau_{\mathrm{DQ}}\right)=D_{\text {res }} k / D_{\text {stat }}$ is model dependent, as it depends on a quasistatic reference value $D_{\text {stat }} / k(\approx 8.1 \mathrm{kHz}$ for cis-PB $)$ defined by the monomer-specific spin system and a realistic model for fast subsegmental dynamics [20]. $D_{\text {res }}$ for a cis unit is obtained from a two-parameter fit according to [16]

$$
\begin{aligned}
I_{\mathrm{nDQ}}= & \exp \left\{-2 \tau_{\mathrm{DQ}} / T_{2}^{\mathrm{app}}\right\}\left[0.25\left(1-\exp \left\{-\frac{2}{5} D_{\mathrm{res}}^{2} \tau_{\mathrm{DQ}}^{2}\right\}\right)\right. \\
& \left.+0.25\left(1-\exp \left\{-\frac{8}{5} D_{\mathrm{res}}^{2} \tau_{\mathrm{DQ}}^{2}\right\}\right)\right],
\end{aligned}
$$

reflecting equal contents of $c$ is and trans units, which differ in $D_{\text {res }}$ by a factor of about 2 [12]. With $D_{\text {res }}=100 \pm$ $30 \mathrm{~Hz}$ [dashed line in Fig. 1(a)], we obtain $C\left(\tau_{\mathrm{DQ}} \approx\right.$ $1 \mathrm{~ms}) \approx 0.00015$, fixing $A$ in Eq. (1). Anticipating the results below, this translates into $C\left(\tau_{e}\right) \approx 2 \times 10^{-3}$, therefore $S_{e} \approx 4.5 \%$, and $N_{e} \approx 13$. This is in reasonable agreement with the literature value of 17 [19], supporting the
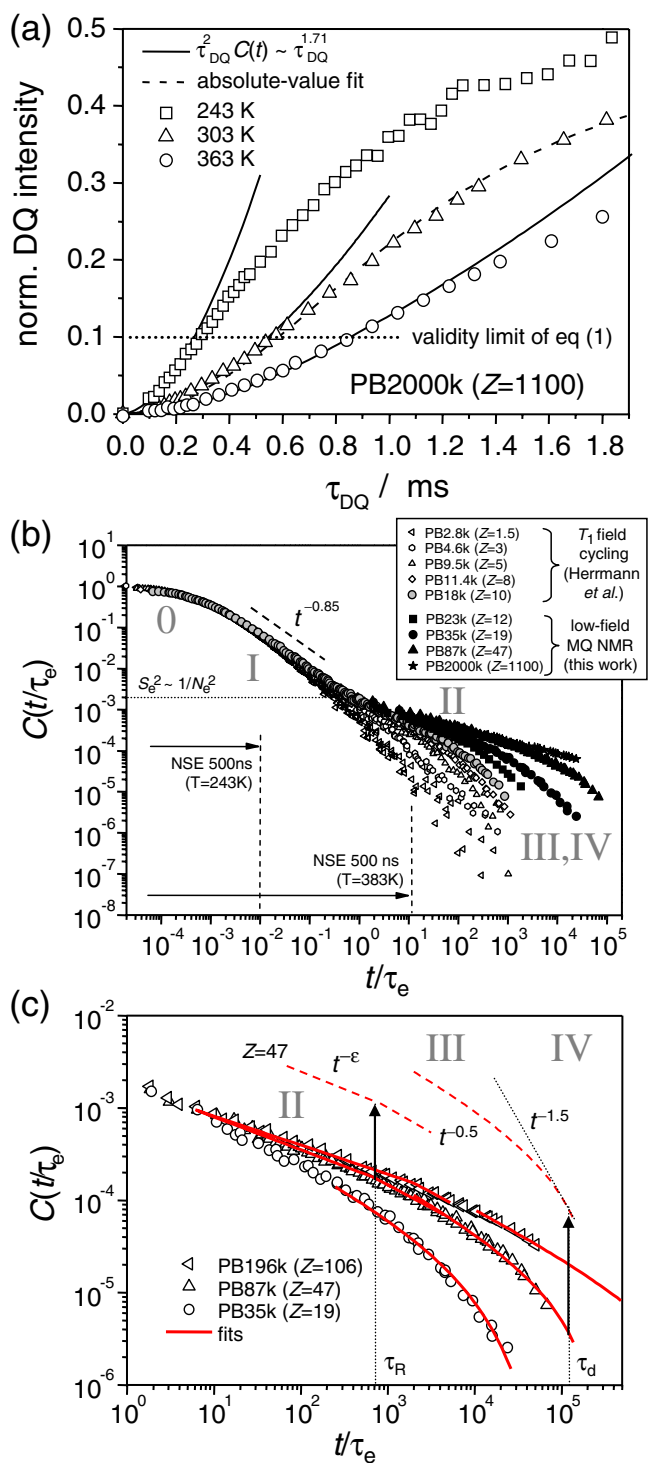

FIG. 1 (color online). (a) Normalized DQ buildup curves of PB2000k at three different temperatures, on a scale relative to the total signal of entangled segments, taken on a Bruker minispec mq20 low-field spectrometer. Dashed line: fit to determine the absolute value of $C(t)$, see text. Solid lines: Power laws $\sim \tau_{\mathrm{DQ}}^{2} C\left(\tau_{\mathrm{DQ}}\right) \sim \tau_{\mathrm{DQ}}^{1.71}$, demonstrating the validity range of Eq. (1). (b) Selected correlation functions $C\left(t / \tau_{e}\right)$ measured in this work, combined with field-cycling NMR data from Ref. [15] covering the lower time range, with no relative shifts applied. The dashed lines indicate the time limits for complementary NSE spectroscopy in the studied temperature interval. (c) $C\left(t / \tau_{e}\right)$ for three samples, with fits used to extract $\tau_{R}, \tau_{d}$, and the scaling exponent $\epsilon$. Dashed lines are the offset fits for PB87k with indicated regime transitions.

model for $D_{\text {stat }} / k$ for which an uncertainty of $\sim 40 \%$ is realistic [20]. Much larger errors related to their intensity reference were probably the reason for the unexpectedly high local order reported in Ref. [12], which was subject to many controversial discussions.

In order to construct $C\left(t / \tau_{e}\right) \propto I_{\mathrm{nDQ}}\left(\tau_{\mathrm{DQ}}\right) / \tau_{\mathrm{DQ}}^{2}$, we perform time-temperature superposition (TTS) using the 
$T$ dependence of the friction coefficient $\zeta(T)$ [21]. Obviously, the initial-rise approximation represented by Eq. (1) is valid only for intensities $I_{\mathrm{nDQ}} \leq 10 \%$ [see dotted line in Fig. 1(a)], which means that only the early part of the curves can be used. By combining data taken at different $\tau_{\mathrm{DQ}}$ and temperatures, the envelope $C(t)$ is easily obtained. All time scales are referenced to $\tau_{e}(T) \approx$ $\zeta(T) N_{e}^{2} b^{2} / \mathrm{kT} \approx \tau_{s}(T) N_{e}^{2}$ [1] with $b \approx 1 \mathrm{~nm} \mathrm{[19].}$

In Fig. 1(b), our data are first compared with the previously published field-cycling results of Rössler and coworkers [15]. The agreement and mutual complementarity of the two methods is near perfect, constituting a molecular measure of the chain dynamics over more than 10 effective decades. Current computer simulations cover roughly the same time range as is accessible by field cycling NMR, and good agreement between experimental and simulated $C(t)$ could be reported $[15,22]$. MQ NMR picks up where fieldcycling NMR experiences problems due to the difficult measurement of $T_{1}$ at low magnetic fields, at which earth and stray field compensation is an issue. We emphasize that this is an absolute-value comparison along both axes. Only the scale of the $x$ axes was matched; they are both referenced to $\tau_{s} \approx 13 \tau_{\alpha}$, with the factor arising from a simple comparison of respective literature data [21,23].

Field-cycling NMR further covers the same time range as NSE, making these methods perfectly complementary. A joint analysis should provide more information on the initial regimes, where for instance, Rössler et al. interpret the slope of -0.85 in regime I in terms of a limited number of possible Rouse modes in an entangled chain section [15]. However, different intra- and intermolecular contributions to $T_{1}$ may also be relevant [14]. Recent theoretical work actually supports that topological constraints even modify the very local chain dynamics [24]. Further, it is still not clear how $\tau_{s}$ and $\tau_{\alpha}$ are related, posing the question of the size and the properties of the "Rouse unit" $[13,25]$. Low-field NMR now complements both methods towards a time range well into regime IV.

In Fig. 1(c), sample data are shown in a log-log representation, along with fits to locate the crossover times $\tau_{R}$ and $\tau_{d}$, using a fit with two connected straight lines of fixed slope for the former, and the analytical result of Ball et al. [17] for the latter. The good fitting quality confirms the validity of this theory in regimes III and IV, which has not been put to a direct test before. Importantly, above $\tau_{e}$ we identify an extended regime II with a constant slope $\epsilon$ characterizing the flattest region of $C(t)$. It is constant over decades in time, as confirmed by derivative analysis, and is reliably determined independently of $\tau_{R}$. The $M$ dependence of $\epsilon$ represents the major finding of this work, with a value of $1 / 2$ for a chain as long as $Z \approx 10$, and never reaching the predicted value of $1 / 4$ within our $M$ range.

Results of the fits are compiled in Figs. 2(a) and 2(b), where the error bars indicate the confidence intervals given by the least-squares fitting routine. Within our error margin, the molecular weight dependence of $\tau_{R}$ agrees with the
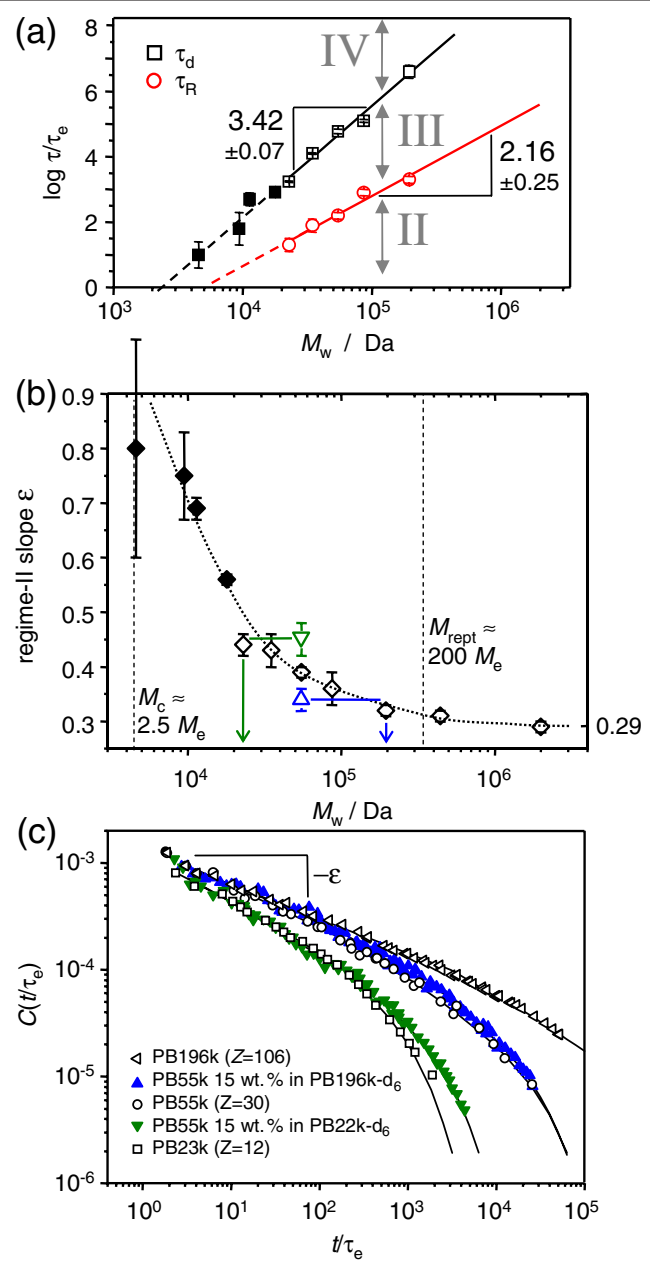

FIG. 2 (color online). (a) $M$ dependence of $\tau_{R}$ and $\tau_{d}$, and (b) the regime-II (constrained-Rouse) scaling exponent $\epsilon$. Raw data from field-cycling NMR [15], solid symbols, was reanalyzed using our fitting procedures. The dotted line for $\epsilon$ is merely a guide to the eye, demonstrating a very protracted approach of a value larger than the tube-model prediction of $1 / 4$. The critical molecular weights $M_{c}$ for entanglement effects on viscosity, and $M_{\text {rept }}$ above which viscosity scales according to the reptation model, $\eta \sim M^{3}$ [26], are indicated. (c) $C\left(t / \tau_{e}\right)$ for PB55k diluted to 15 wt. \% in different deuterated PBs. The respective exponents $\epsilon$ plotted as triangles in (b) are demonstrated to be dependent on the matrix chains, indicated by arrows. The pure $\mathrm{PB}$ reference data were multiplied by 0.75 to account for lower overall values due to missing interchain dipolar couplings. The factor was determined for PB196k diluted in the same deuterated $M$, which only lead to a vertical shift of the whole $C\left(t / \tau_{e}\right)$ without changes in its shape.

tube-model prediction, while the scaling for $\tau_{d}$ displays the well-documented $M^{3.4}$ dependence that is commonly observed for the melt viscosity or the terminal relaxation time from rheological experiments [26], as long as the chain length is below $Z \approx 200$. The +0.4 deviation from the ideal (fixed-tube) reptation scaling has been attributed to $\mathrm{CR}$ effects for the special case of dielectric experiments on poly(isoprene) probe chains in miscible blends with $\mathrm{PB}$ matrix chains [6], but the relevance of either CR or CLF 
effects on the terminal relaxation in a homopolymer melt is the subject of ongoing discussion $[4,5,26]$. We take the observed $\tau_{R}$ and $\tau_{d}$ scalings with $M$ as a confirmation of the validity of TTS, yet we note that a consistent $\epsilon(T)$ can also be obtained directly from the initial $I_{\mathrm{nDQ}}\left(\tau_{\mathrm{DQ}}\right)$ at fixed $T$. Details on our data analysis will be published shortly.

The $M$ dependence of the regime-II scaling exponent $\epsilon$ reflects deviations from the picture of a fixed-tube constraining nonglobal chain motion (slower Rouse modes) before reptation sets in. Below $M_{c}$ we consistently observe a merging of $\epsilon$ into the range typical for regime I. At high $M$, where $\epsilon$ is still measured accurately and transitions to regimes III and IV cannot even be reached due to sample degradation at high temperature, we notice a smooth transition to values approaching but not quite reaching the prediction of $1 / 4$, thus indicating that a static tube constraint is hardly realized. These findings do not appear compatible with current single-chain mode based theories, highlighting, in particular, the limitations of the fixed-tube reptation model. Our regime assignments must therefore be considered preliminary and subject to a refined analysis. We note that our lowest $\epsilon$ value of 0.29 is close to the limiting exponent $9 / 29=0.281$ for the time scaling of $\left\langle\Delta r^{2}\right\rangle$ predicted by polymer mode coupling theory [27]. However, it remains to be shown whether the return-toorigin arguments used in Refs. [2,17], which convert the tube-model prediction $\left\langle\Delta r^{2}\right\rangle \sim t^{1 / 4}$ into the intuitive inverse $C(t) \sim t^{-1 / 4}$ law, are also applicable in this case.

Irrespective of the validity of different entanglement theories, we can directly prove the major influence of CR effects by studying mixtures with ${ }^{2} \mathrm{H}$-labeled components in analogy to previous investigations [6,7], where changes in the dynamics of dilute probe chains were observed by changing the length of invisible matrix chains. In Fig. 2(c), we show correlation functions for PB55k diluted in a lower- $M$ and a higher- $M$ matrix, and the results also plotted in Fig. 2(b) immediately demonstrate that $\epsilon$ of the probe chain corresponds to that of the pure matrix chains. As to the overall changes in $C\left(t / \tau_{e}\right)$, we find that the only significant effect of the higher- $M$ matrix is the change in $\epsilon$, while $C R$ in a lower- $M$ matrix slaves the terminal dynamics of the probe chain and changes the shape of $C\left(t / \tau_{e}\right)$ completely. Our results complement recent NSE experiments probing regime $0-$ II motions, where significant $\mathrm{CR}$ effects could only be studied by diluting longer chains in a lowly entangled matrix [7].

In summary, we have established the robust measurement of the orientation autocorrelation function $C(t)$ as a direct probe of entangled chain dynamics, revealing limitations of the tube model. Focusing on $C(t)$ alongside the common prediction of dynamic structure factors, future theory work will be aided by our results in identifying proper models for entanglement constraints that can correctly reflect our data. Ongoing work is dedicated to a study of regime transition times for different probe chains in even longer matrices, where we expect further evidence on the origin of the $M$ scaling of $\tau_{d}$ in homopolymer melts. Additional insights will arise from MQ NMR experiments on ${ }^{2} \mathrm{H}-{ }^{1} \mathrm{H}-{ }^{2} \mathrm{H}$ pseudotriblock copolymers, suppressing signals from chain-end sections, clarifying possible changes in $C(t)$ along the chain.

Funding was provided by the DFG (SA 982/3-1). We are indebted to R. Graf for ongoing advice, and thank E. Straube, E. A. Rössler, and A. Herrmann for valuable discussions, and the latter two for providing their raw data.

*kay.saalwaechter@physik.uni-halle.de

[1] M. Doi and S.F. Edwards, The Theory of Polymer Dynamics (Clarendon Press, Oxford, 1986).

[2] P. G. de Gennes, J. Chem. Phys. 55, 572 (1971).

[3] T. C. B. McLeish, Adv. Phys. 51, 1379 (2002).

[4] C.-Y. Liu, R. Keunings, and C. Bailly, Phys. Rev. Lett. 97, 246001 (2006).

[5] D. J. Read, K. Jagannathan, and A.E. Likhtman, Macromolecules 41, 6843 (2008).

[6] K. Adachi et al., Macromolecules 28, 3588 (1995).

[7] M. Zamponi et al., Phys. Rev. Lett. 96, 238302 (2006).

[8] D. S. Pearson, L. J. Fetters, W. W. Graessley, G. V. Strate, and E. von Meerwall, Macromolecules 27, 711 (1994).

[9] S. Pahl, G. Fleischer, F. Fujara, and B. Geil, Macromolecules 30, 1414 (1997).

[10] M. E. Komlosh and P. T. Callaghan, J. Chem. Phys. 109, 10053 (1998).

[11] E. Fischer, R. Kimmich, N. Fatkullin, and G. Yatsenko, Phys. Rev. E 62, 775 (2000).

[12] R. Graf, A. Heuer, and H. W. Spiess, Phys. Rev. Lett. 80, 5738 (1998).

[13] S. Kariyo et al., Phys. Rev. Lett. 97, 207803 (2006).

[14] M. Kehr, N. Fatkullin, and R. Kimmich, J. Chem. Phys. 127, 084911 (2007).

[15] A. Herrmann, V.N. Novikov, and E.A. Rössler, Macromolecules 42, 2063 (2009).

[16] K. Saalwächter, Prog. Nucl. Magn. Reson. Spectrosc. 51, 1 (2007).

[17] R. C. Ball, P. T. Callaghan, and E. T. Samulski, J. Chem. Phys. 106, 7352 (1997).

[18] R. Kimmich, M. Köpf, and P. Callaghan, J. Polym. Sci. B 29, 1025 (1991).

[19] Physical Properties of Polymers Handbook, edited by J. E. Mark (Springer, New York, 2007).

[20] K. Saalwächter, B. Herrero, and M. A. López-Manchado, Macromolecules 38, 9650 (2005).

[21] M.-H. Klopffer, L. Bokobza, and L. Monnerie, Polymer 39, 3445 (1998).

[22] T. Kreer et al., Macromolecules 34, 1105 (2001).

[23] J. Hintermeyer et al., Macromolecules 41, 9335 (2008).

[24] F. Lahmar, C. Tzoumanekas, D. N. Theodorou, and B. Rousseau, Macromolecules 42, 7485 (2009).

[25] Y. Ding, A. Kisliuk, and A. P. Sokolov, Macromolecules 37, 161 (2004).

[26] M. Abdel-Goad et al., Macromolecules 37, 8135 (2004).

[27] K. S. Schweizer et al., Macromol. Theory Simul. 6, 1037 (1997). 